\title{
cAMP-Dependent Regulation of RhoA/Rho-kinase Attenuates Detrusor Overactivity in a Novel Mouse Experimental Model
}

\author{
William Akakpo, Biljana Musicki, and Arthur L. Burnett \\ The James Buchanan Brady Urological Institute and Department of Urology, The Johns Hopkins \\ School of Medicine, Baltimore, Maryland, United States of America
}

\begin{abstract}
Objectives-To investigate detrusor function and cAMP activation as a possible target for detrusor overactivity in an experimental model lacking a key denitrosylation enzyme, Snitrosoglutathione reductase (GSNOR).
\end{abstract}

\begin{abstract}
Materials and Methods-GSNOR-deficient $\left(\mathrm{GSNOR}^{-/}\right)(\mathrm{n}=30)$ and wild-type (WT) mice $(\mathrm{n}=26)$ were treated for 7 days with the cAMP activator, colforsin $(1 \mathrm{mg} / \mathrm{kg})$, or vehicle intraperitoneally. Cystometric studies or molecular analyses of bladder specimens were performed. Bladder function indices and expression levels of proteins that regulate detrusor relaxation (nitric oxide synthase pathway) or contraction (RhoA/Rho-kinase pathway) and oxidative stress were assessed. Student t-test and one-way ANOVA were used.
\end{abstract}

Results- $\mathrm{GSNOR}^{-/-}$mice showed a significant increase $(\mathrm{P}<0.05)$ in voiding and non-voiding contraction frequencies compared to WT mice (Cohen's effect size values $\mathrm{d}=1.82$ and 2.52, respectively). Colforsin normalized these abnormalities (Cohen's effect size values $d=1.85$ and 1.28 , respectively). Western blot analyses showed an up-regulation of the RhoA/Rho-kinase pathway reflected by a significant increase $(\mathrm{P}<0.05)$ of phosphorylated-MYPT1 expression in $\mathrm{GSNOR}^{-/-}$mouse bladders, which was reversed by colforsin treatment. An increased level $(\mathrm{P}<0.05)$ of gp91 ${ }^{\text {phox }}$ expression in bladders of $\mathrm{GSNOR}^{-/}$mice was observed without significant change after colforsin treatment. Neuronal and endothelial nitric oxide synthase phosphorylation on Ser-1412 and Ser-1177, respectively, did not differ between GSNOR $^{-/}$and WT mouse bladders irrespective of colforsin treatment.

Conclusion-Impaired denitrosylation is associated with detrusor overactivity that is linked with upregulated RhoA/Rho-kinase signaling. Colforsin reverses physiologic and molecular abnormalities. This study describes a novel model of detrusor overactivity and suggests a possible basis for its treatment.

\footnotetext{
Corresponding author: Arthur L. Burnett, MD, MBA, The James Buchanan Brady Urological Institute and Department of Urology, The Johns Hopkins School of Medicine, 601 North Caroline Street, Baltimore, Maryland, 21205, United States of America, Tel: +1 410614 3986, Fax: +1 410614 3695, aburnet1@jhmi.edu.

Conflicts of Interest

None
} 


\section{Introduction}

The bladder has two physiologic functions, urine storage and release, which describes its cyclic relaxation and contraction. These mechanisms work through an interaction between the nervous system and the urothelium and smooth muscle of the bladder [1]. The urothelium plays a sensory role and releases mediators to modulate smooth muscle tone [2]. Deregulation of mechanisms controlling contraction and relaxation can lead to bladder dysfunction such as detrusor overactivity [3]. Nitric oxide is considered to contribute to regulation of detrusor tone, but its role in the bladder remains controversial. Reduction in NO level in the bladder using NO synthase (NOS) inhibitors or mice with targeted deletion of the gene for neuronal NOS results in bladder overactivity or a dilated hypertrophic bladder phenotype, respectively $[4,5]$. However, NO donors have also been reported to cause small or inconsistent relaxations in the rat and mouse detrusor [6, 7], and a complex response (relaxation and contraction) in pre-contacted human detrusor [8], indicating that the role of NO in the bladder is still incompletely understood.

Several mechanisms are involved in protein activity regulation. S-nitrosylation, which is a post-translational modification of a cysteine residue on target proteins by NO, is a major mechanism affecting NO responses [9]. A wide variety of proteins are activated or inactivated by S-nitrosylation. This modification is regulated by S-nitrosoglutathione reductase (GSNOR), which mediates the denitrosylation process. Excessive S-nitrosylation plays a role in many human diseases such as diabetes mellitus and pre-eclampsia [10].

An analysis of the GSNOR deficient $\left(\mathrm{GSNOR}^{-/-}\right)$mouse penis showed increased levels of S-nitrosylated proteins, reduced NO bioavailability, endothelial NOS (eNOS) dysfunction, and increased oxidative damage, and these mice are predisposed to erectile dysfunction [11]. S-nitrosylation and its deregulation may also affect bladder homeostasis due to NO functional impairment. However, data remain poorly documented in this field.

We hypothesized that the bladder behaves similarly to the penis, both sharing common counter-regulatory mechanisms responsible for tissue relaxation and contraction involving NOS and RhoA/Rho-kinase pathways, respectively. The NOS pathway has been widely studied in the penis and plays a central role in erection physiology [12]. RhoA/Rho-kinase signaling is understood to modulate erectile tissue contraction in the penis [13] and to be involved in molecular mechanisms of the obstructed bladder [14]. Accordingly, we used $\mathrm{GSNOR}^{-/-}$mice to characterize bladder function, the aforementioned mediators and oxidative stress in the context of deregulated S-nitrosylation. Our hypothesis was that $\mathrm{GSNOR}^{-/-}$mice exhibit bladder function abnormalities due to NOS and RhoA/Rho-kinase pathway deregulations. We conjectured that mechanisms controlling urinary bladder smooth muscle relaxation and contraction represented respectively by NOS and RhoA/Rho-kinase pathways are deranged in the context of deregulated S-nitrosylation, leading to bladder dysfunction. We also assessed the effect of cAMP activation, which has a dual effect on neuronal and endothelial NOS activation and RhoA/Rho-kinase inhibition [15, 16], in this experimental model. 


\section{Materials and Methods}

\section{Animals}

Adult male (3.5 months old) homozygous $\mathrm{GSNOR}^{-/-}$and age-matched wild-type (WT) mice (C57BL/6, The Jackson Laboratory, Bar Harbor, ME, USA) were used. All experiments were approved by the Animal Care and Use Committee of the Johns Hopkins University School of Medicine and were conducted in accordance with the National Institutes of Health Guide for the Care and Use of Laboratory Animals.

\section{Study design}

$\mathrm{GSNOR}^{-/-}(\mathrm{n}=30)$ and WT $(\mathrm{n}=26)$ mice were treated for seven days with colforsin daparoate (Abcam, Cambridge, UK) at $1 \mathrm{mg} / \mathrm{kg} /$ day intraperitoneally [17] or vehicle (saline). Colforsin is a water soluble analogue of forskolin that activates adenylyl cyclase to produce cAMP [18]. Forskolin was described to induce smooth muscle relaxation in urinary bladder from streptozotocin-treated mice [19]. We favoured colforsin rather than forskolin because of its water solubility to avoid DMSO toxicity [20]. Moreover, colforsin has no effect on blood pressure (Karakus S, Musicki B, La Favor J, Burnett AL, unpublished work). 24 hours after the last injection of colforsin or vehicle, mice were used for cystometry or bladder collection for Western blot analyses.

\section{Cystometry}

24 mice (n=six/group) were anesthetized by intraperitoneal injection of urethane $(1.8 \mathrm{mg} /$ $\mathrm{kg}$ ), and a laparotomy was done to expose the bladder. The bladder was emptied and a 25gauge butterfly needle was inserted into the bladder dome. This needle was connected to a two-way tap, of which one port was connected to a pressure transducer and the other was connected to an infusion pump. Continuous cystometry was performed during 30 minutes by infusing saline in the bladder at $10 \mu \mathrm{l}$ per minute. The parameters assessed were baseline pressure (lowest pressure between voids), threshold pressure (pressure at which voiding was initiated), peak pressure (maximum pressure observed during a micturition cycle), bladder capacity (maximum volume infused before urine expulsion, assessed at the end of the cystometry), compliance (change in volume/change in pressure), and the frequency of voiding and non-voiding contractions (NVCs). Voiding contraction was defined as a bladder pressure increase accompanied by saline expulsion from the urethra (micturition). Nonvoiding contraction was not accompanied by micturition [21, 22]. Cystometry data were recorded using the DI-190 system (Dataq Instruments, Akron, OH, USA).

\section{Western Blot Analysis}

32 mice (7 WT + vehicle, $7 \mathrm{WT}$ + colforsin, $9 \mathrm{GSNOR}^{-1-}$ + vehicle, and $9 \mathrm{GSNOR}^{-1-}$ + colforsin) were anesthetized (100 mg/kg Ketamine $+10 \mathrm{mg} / \mathrm{kg}$ Xylazine, intraperitoneally) for bladder collection. Bladders were immediately snap frozen in liquid nitrogen and homogenized, as described previously [23]. Bladder homogenates were resolved on $7.5 \%$ or $4-20 \%$ Tris gels and transferred to polyvinylidene difluoride membranes. Membranes were probed with polyclonal anti-phospho-endothelial NOS (P-eNOS) on Ser-1177 antibody (Cell Signaling Technology, Beverly, MA, catalog number 9571S) at 1:450, polyclonal anti- 
phospho-neuronal NOS (P-nNOS) on Ser-1412 antibody (gift from Dr. Solomon Snyder, Johns Hopkins Hospital, Baltimore, MD, USA) at 1:6000, polyclonal anti-phospho-MYPT-1 (P-MYPT-1) on Thr-696 antibody at 1:1000 (Cell Signaling Technology, catalog number 5163), monoclonal anti-Rho-kinase (ROCK) I antibody (Santa Cruz Biotechnology, Dallas, TX, USA, catalog number sc-17794) at 1:500, monoclonal anti-ROCK II antibody (BD Transduction Laboratories, San Diego, CA, catalog number 6106624) at 1:1000, polyclonal anti-4-hydroxy-2-nonenal (4-HNE) antibody (Alpha Diagnostic International, San Antonio, TX, USA, catalog number HNE-11-S) at 1:5000, monoclonal anti-nitrotyrosine antibody (Abcam Inc, Cambridge, MA, USA, catalog number ab-42789) at 1:2000, and monoclonal anti-gp91 phox antibody (BD Transduction Laboratories, catalog number 611414) at 1:1000 dilutions. These membranes were stripped and probed with antibodies for the index proteins, monoclonal anti-eNOS (BD Transduction Laboratories, catalog number 612392) at 1:500, polyclonal anti-nNOS (gift from Dr. Solomon Snyder) at 1:1000, polyclonal anti-MYPT-1 (Santa Cruz Biotechnology, catalog number sc-25618) at 1:1000, and monoclonal antiglyceraldehyde 3-phosphate dehydrogenase (GAPDH) (Santa Cruz Biotechnology, catalog number sc-32233) at 1:5000 dilutions. Bands were detected by horseradish peroxidase conjugated antibodies (GE Healthcare, Piscataway, NJ, USA, catalog numbers NA931V and NA934V) and analyzed using NIH Image software. P-eNOS, P-nNOS, and P-MYPT-1 densities were normalized relative to that of eNOS, nNOS, and MYPT-1, respectively. Other signals (ROCK1, ROCK2, gp91phox, 4-HNE, nitrotyrosine, nNOS, and eNOS) were standardized to GAPDH. Results were expressed relative to values of WT mouse treated with vehicle. To verify that GAPDH expression was not affected by any of the treatments, the density of GAPDH in a set of samples $(n=9)$ was standardized per total proteins (Ponceau S staining). There was no difference in protein expression of GAPDH in bladders of WT-treated mice or GSNOR-/- mice with or without colforsin treatment (90.01 $\pm 10.08 \% ; 81.33 \pm 17.33 \% ; 94.34 \pm 10.79 \%$, respectively) compared to WT mice treated with vehicle (100\%).

\section{Statistical Analysis}

The sample sizes were not predetermined by statistical methods. Study design was based on experimental methodology and statistics of prior experimental work. Statistical analyses were performed using one-way ANOVA followed by the Tukey for multi-groups analyses of cystometric parameters (Prism v.5, GraphPad Software, San Diego, CA USA). In order to compare samples on Western blots performed on different membranes, we used a modified $t$ test to compare the experimental groups with the normalized control ratio (WT + vehicle). All data were expressed as the mean plus or minus standard error or the mean (SEM) [24]. A value of $\mathrm{P}<0.05$ was considered to be statistically significant [25]. Cystometric parameters results were coupled with effect size measure (Cohen's d) [26].

\section{Results}

\section{Voiding and non-voiding contraction frequencies are increased in $\mathrm{GSNOR}^{-/}$mice and normalized by colforsin treatment}

Cystrometric tracings showed that untreated $\mathrm{GSNOR}^{-/-}$mice had a greater number of voiding and non-voiding contractions compared to untreated WT mice (Fig.1). Statistical 
analyses showed that untreated $\mathrm{GSNOR}^{-/-}$mice had a significant increase $(\mathrm{P}<0.05)$ in both voiding and non-voiding contraction frequencies compared to WT mice (Fig. 2). Further, Cohen's effect size values ( $\mathrm{d}=1.82$ and 2.52 , respectively) suggested high practical significance. There was no significant difference between GSNOR-/- and WT mice for the other cystometric parameters. GSNOR ${ }^{-/-}$mice treated with colforsin showed significant decreases $(\mathrm{P}<0.05)$ in both voiding and non-voiding contraction frequencies compared to that of $\mathrm{GSNOR}^{-/-}$mice treated with vehicle, and Cohen's effect size values ( $\mathrm{d}=1.85$ and 1.28 , respectively) suggested high practical significance. We did not observe a drug effect on cystometric parameters of WT mice.

\section{Phosphorylation of nNOS (Ser-1412) and eNOS (Ser-1177) is not changed in the $\mathrm{GSNOR}^{-/-}$ mouse bladder and is not affected by colforsin treatment}

There was no significant difference in the protein expressions of P-nNOS (Ser-1412), PeNOS (Ser-1177), nNOS and eNOS in bladders of untreated $\mathrm{GSNOR}^{-1}$ compared to WT mice (Fig. 3). There was also no significant difference in these protein expression levels after colforsin treatment. Standardization of treatment groups per control did not allow us to analyze the absolute magnitude of P-eNOS (Ser-1177) changes in a $\mathrm{GSNOR}^{-/-}$mouse bladder by colforsin treatment.

\section{Protein expression of P-MYPT-1 (but not ROCK I and ROCK II) is increased in the GSNOR $^{-/}$mouse bladder and normalized by colforsin treatment}

ROCK I and II protein expression levels were not significantly different between untreated GSNOR $^{-/-}$and WT mouse bladders. However, the ratio of P-MYPT-1 (Thr-696) to total MYPT-1, reflecting RhoA/Rho-kinase activity, was significantly higher $(\mathrm{P}<0.05)$ in bladders of $\mathrm{GSNOR}^{-/-}$mice compared to that of WT mice (Fig. 4). Colforsin reduced significantly $(\mathrm{P}<0.05)$ the protein expression level of P-MYPT-1 to levels similar to the levels found in WT mice without any modification of ROCK I or II expression in bladders of GSNOR ${ }^{-/}$ mice. This treatment did not lead to any significant modification of ROCK I or II and PMYPT-1 expressions in bladders of WT mice.

\section{Protein expression of NADPH oxidase subunit gp91 phox (but not oxidative stress markers) is increased in the GSNOR-/- mouse bladder and not affected by colforsin treatment}

Protein expression of NADPH oxidase catalytic subunit gp91 phox was significantly increased $(\mathrm{P}<0.05)$ in bladders of untreated $\mathrm{GSNOR}^{-/-}$compared to that of WT untreated mice (Fig.

5). However, there was no significant difference in expressions of markers of oxidative stress, 4-HNE and nitrotyrosine, in $\mathrm{GSNOR}^{-/}$compared to WT untreated mouse bladders. There was no significant difference of gp91 ${ }^{\text {phox }}$ expression in bladders of $\mathrm{GSNOR}^{-/-}$mice after treatment with colforsin. There was also no significant difference in 4-HNE and nitrotyrosine expressions in bladders of $\mathrm{GSNOR}^{-/-}$and WT mice after colforsin treatment.

\section{Discussion}

In this study, we demonstrate a pivotal role of S-nitrosylation in bladder homeostasis that is linked with cAMP-regulated RhoA/Rho-kinase signaling of bladder contractility. The absence of regulation of S-nitrosylation in $\mathrm{GSNOR}^{-/-}$mice leads to detrusor overactivity in 
association with upregulated activity of the RhoA/Rho-kinase pathway (Fig. 6). cAMP activation by colforsin reverses bladder dysfunction in $\mathrm{GSNOR}^{-/-}$mice conceivably by inhibiting abnormal RhoA/Rho-kinase signaling.

S-nitrosylation is a physiologically important post-translational modification with functional consequences for signaling protein activity [9]. GSNOR is a key enzyme regulating this modification. To our knowledge, our study is the first to report cystometric findings in $\mathrm{GSNOR}^{-/-}$mice characterized by abnormally increased voiding and non-voiding contraction frequencies, suggestive of detrusor overactivity [27]. This pathologic model allowed us to explore the ameliorative effect of colforsin, a direct activator of adenylate cyclase that increases cAMP production. Also, our molecular studies highlighted two main findings in the $\mathrm{GSNOR}^{-/}$mouse bladder: abnormally increased protein expression levels of P-MYPT-1 (Thr-696) and gp91 ${ }^{\text {phox }}$ subunit.

MYPT-1 is one of the regulatory subunits of myosin light chain phosphatase (MLCP) that dephosphorylates the myosin light chain (MLC) [28]. This dephosphorylation initiates the relaxation process of smooth muscle cells. Conversely, the phosphorylation of MLC by myosin light chain kinase (MLCK) leads to smooth muscle contraction [29]. MLCP activity is regulated through phosphorylation of MYPT1 on Thr-696 by ROCK that inhibits its phosphatase activity and thereby causes smooth muscle contraction [30]. Thus, P-MYPT-1 is a reflection of RhoA/Rho-kinase pathway activity, and its excessive levels in GSNOR ${ }^{-/-}$ mouse bladders conceivably lead to excessive smooth muscle contraction and detrusor overactivity. A previous study demonstrated that carbachol activates the RhoA/Rho-kinase pathway, leading to MYPT-1 phosphorylation at Thr-696 and sustained contraction in rat bladder smooth muscle cells [31]. In our study, basal expression levels of the RhoA/Rhokinase pathway were unchanged, but the increased level of phospho-MYPT-1 is in line with increased activity of this pathway. S-nitrosylation has been reported to regulate the activity of many enzymes including kinases and in the experimental context of $\mathrm{GSNOR}^{-/-}$mice, ROCK is conceivably nitrosylated as a consequence of deregulated denitrosylation [32]. It is known that in many conditions such as ageing, RhoA/Rho-kinase activity is increased but its constituent expression levels are normal [33]. The implication of RhoA/Rho-kinase pathway derangement in this pathologic model supports the hypothesis that the bladder and penis share common mechanisms of regulation. In fact, RhoA/Rho-kinase signaling deregulation is also associated with erectile dysfunction $[34,35]$.

Gp91 ${ }^{\text {phox }}$ is a subunit of NAPDH oxidase, which is a major source of reactive oxygen species like superoxide. Bladders of $\mathrm{GSNOR}^{-/-}$mice expressed an increased level of this subunit without any effect by colforsin. Evidence is lacking to affirm that increased gp91 phox expression is the consequence of lack of denitrosylation, but it is known that $\mathrm{p} 47^{\mathrm{phox}}$, another subunit of NADPH oxidase, is regulated by S-nitrosylation [36]. Increased NADPH oxidase activity is understood to contribute to oxidative stress. However, 4-HNE and nitrotyrosine, which are biomarkers for oxidative stress and described to be increased in the $\mathrm{GSNOR}^{-1-}$ mouse penis, were normal in the $\mathrm{GSNOR}^{-/-}$mouse bladder. One possible explanation for this finding is that gp91 $1^{\text {phox }}$ does not contribute to NADPH oxidase activity in the bladder. In fact, it was described using gp91 ${ }^{\text {phox }}$-deficient mice that gp91 ${ }^{\text {phox }}$ does not contribute to NADPDH oxidase activity in aortic smooth muscle cells [37]. The fact that 
oxidative stress markers, 4-HNE and nitrotyrosine, are normal here suggests that this elevation of gp91 ${ }^{\text {phox }}$ may have a less likely impact on NADPH oxidase activity and consequently oxidative stress in the bladder. Moreover, it was described that NADPH oxidase gene expression is modulated by cAMP-dependent protein kinase A (PKA) in microglia and neurons [38]. Our interpretation that $\mathrm{gp} 91^{\mathrm{phox}}$ is not an accurate marker of NADPH oxidase activity in the bladder fits with the absence of modification of gp91 phox protein expression after cAMP activation by colforsin treatment. However, further screening of other oxidative stress markers and superoxide itself may be useful to affirm this assessment.

Protein expressions of both endothelial and neuronal isoforms of NOS and their phosphorylated isoforms (P-eNOS on Ser-1177 and P-nNOS on Ser-1412, respectively) were not significantly different in $\mathrm{GSNOR}^{-/}$and WT mouse bladders. These phosphorylation sites play major mediatory roles in penile erection. We conjectured first that they are also important in bladder function, although this was not shown here. However, other NOS isoform phosphorylation sites may be involved as a result of RhoA/Rho-kinase pathway upregulation. Activation of RhoA/Rho-kinase signaling inhibits eNOS through phosphorylation on Thr-495, which dramatically decreases the levels of NO production leading to an increase of vascular tone [39]. It is also possible that NO levels are directly affected by S-nitrosylation of downstream effectors such as phosphodiesterase or soluble guanylyl cyclase (sGC) [40]. This situation is associated with normal levels of NOS isoforms but possibly abnormal NO signaling contributing to bladder overactivity.

The central role of cAMP formation in the penis and its relationship with RhoA/Rho-kinase pathway signaling [41] prompted us to use colforsin. cAMP activation has been considered to play a role in smooth relaxation involving $\beta$-adrenoceptors by reducing both calcium mobilization and sensitivity of the contractile machinery to calcium [42]. However, a definitive role of cAMP-dependent $\beta$-adrenoceptor function remains uncertain. Its inhibition appears to have no effect on human detrusor function [43]. Nevertheless, we employed a 24hour wash-out period to prevent this controversial relaxant effect and sought to elicit changes in specific mechanisms modulating the function of NOS and RhoA/Rho-kinase pathways. While its effect on NOS was neutral, this drug appeared to normalize RhoA/Rhokinase activity as determined by P-MYPT-1 (Thr-696) measurement. Thus, the unphosphorylated and active form of MLCP dephosphorylates MLC and leads to bladder relaxation. The main mechanism of action of colforsin conceivably involves the upstream mediator RhoA. The phosphorylation of RhoA on Ser- 188 by cAMP-dependent PKA, inducing its inhibition and consequently RhoA/Rho-kinase activity inhibition, leads to the normalization of P-MYPT-1 levels [44, 45]. Furthermore, it was reported that PKA activation may inhibit protein S-nitrosylation [46]. This complementary effect may also contribute to the beneficial impact of colforsin.

We acknowledge several possible limitations of our work. First, despite showing that MYPT-1 phosphorylation, as a reflection of RhoA/Rho-kinase activity was increased in the $\mathrm{GSNOR}^{-/-}$mouse bladder, we did not measure Rho-kinase activity directly to validate our hypothesis regarding the upregulation of RhoA/Rho-kinase [47]. It would also have been worthwhile to assess the effect of a Rho-kinase inhibitor such as fasudil on bladder function 
in our experimental model [48] however, the effectiveness of RhoA/Rho-kinase inhibition was previously described to reverse acetic acid-induced bladder overactivity in rats [49]. Furthermore, although we did not find any change in NOS phosphorylation (P-nNOS on Ser1412 and P-eNOS on Ser-1177), it remains possible that other phosphorylation sites on nNOS and eNOS are involved in bladder function. To clarify better these mechanisms, further studies in the bladder with analyses of other NOS phosphorylation sites and total NO levels may be done. Also, nitrosylation measurements of regulatory proteins such as ROCK isoforms and $\mathrm{sGC}$ are warranted in order to establish a cause-effect relationship between denitrosylation and bladder function. Considering the physiologic role of the entire lower genitourinary tract, additional molecular studies may extend to the urethra. Finally, the systemic effects of colforsin, in particular cardiovascular effects, should be assessed to validate its safe use.

In conclusion, this study reported for the first time, to our knowledge, an experimental model of detrusor overactivity related to GSNOR deficiency. The putatively decreased denitrosylation of ROCK leads to its upregulated activity and bladder overactivity which we observed physiologically. This bladder dysfunction was reversed by cAMP activation, which inhibits RhoA/Rho-kinase signaling. These findings contribute to understanding the pathophysiology of bladder overactivity and may suggest a novel potential therapeutic approach.

\section{Acknowledgments}

R01DK067223, DR. WILLIAM AKAKPO (Orcid ID : 0000-0003-1683-0079)

\section{References}

1. de Groat WC, Griffiths D, Yoshimura N. Neural control of the lower urinary tract. Compr Physiol. 2015; 5:327-96. [PubMed: 25589273]

2. Birder L, Andersson KE. Urothelial signaling. Physiol Rev. 2013; 93:653-80. [PubMed: 23589830]

3. Steers WD. Pathophysiology of overactive bladder and urge urinary incontinence. Rev Urol. 2002; 4:7-18.

4. Mónica FZ, Bricola AA, Báu FR, et al. Long-term nitric oxide deficiency causes muscarinic supersensitivity and reduces beta(3)-adrenoceptor- mediated relaxation, causing rat detrusor overactivity. Br J Pharmacol. 2008; 153:1659-68. [PubMed: 18297104]

5. Burnett AL, Calvin DC, Chamness SL, et al. Urinary bladder-urethral sphincter dysfunction in mice with targeted disruption of neuronal nitric oxide synthase models idiopathic voiding disorders in humans. Nat Med. 1997; 3:571-4. [PubMed: 9142130]

6. Persson K, Igawa Y, Mattiasson A, Andersson K-E. Effects of inhibition of the 1-arginine/nitric oxide pathway in the rat lower urinary tract in vivo and in vitro. Br J Pharmacol. 1992; 107:178-84. [PubMed: 1422571]

7. Satake Y, Satoh K, Nogi M, et al. Crucial roles of nitric oxide synthases in $\beta$-adrenoceptor-mediated bladder relaxation in mice. Am J Physiol Renal Physiol. 2017; 312:33-42.

8. Moon A. Influence of nitric oxide signalling pathways on pre-contracted human detrusor smooth muscle in vitro. BJU Int. 2002; 89:942-9. [PubMed: 12010245]

9. Stamler JS, Simon DI, Osborne JA, et al. S-nitrosylation of proteins with nitric oxide: synthesis and characterization of biologically active compounds. Proc Natl Acad Sci U S A. 1992; 89:444-8. [PubMed: 1346070]

10. Foster MW, McMahon TJ, Stamler JS. S-nitrosylation in health and disease. Trends Mol Med. 2003; 9:160-8. [PubMed: 12727142] 
11. Musicki B, Lagoda G, Goetz T, La Favor JD, Burnett AL. Transnitrosylation: A Factor in Nitric Oxide-Mediated Penile Erection. J Sex Med. 2016; 13:808-14. [PubMed: 27114194]

12. Burnett AL, Lowenstein CJ, Bredt DS, Chang TS, Snyder SH. Nitric oxide: a physiologic mediator of penile erection. Science. 1992; 257:401-3. [PubMed: 1378650]

13. Wang H, Eto M, Steers WD, Somlyo AP, Somlyo AV. RhoA-mediated Ca2+ sensitization in erectile function. J Biol Chem. 2002 Aug.277:30614-21. [PubMed: 12060659]

14. Bing W, Chang S, Hypolite JA, et al. Obstruction-induced changes in urinary bladder smooth muscle contractility: a role for Rho kinase. Am J Physiol Renal Physiol. 2003; 285:990-7.

15. Russo I, Doronzo G, Mattiello L, De Salve A, Trovati M, Anfossi G. The activity of constitutive nitric oxide synthase is increased by the pathway cAMP/cAMP-activated protein kinase in human platelets. New insights into the antiaggregating effects of cAMP-elevating agents. Thromb Res. 2004; 114:265-73. [PubMed: 15381390]

16. Naviglio S, Caraglia M, Abbruzzese A, et al. Protein kinase A as a biological target in cancer therapy. Expert Opin Ther Targets. 2009; 13:83-92. [PubMed: 19063708]

17. Oishi H, Takano K, Tomita K, Takebe M, Yokoo H, Yamazaki M, Hattori Y. Olprinone and colforsin daropate alleviate septic lung inflammation and apoptosis through CREB-independent activation of the Akt pathway. Am J Physiol Lung Cell Mol Physiol. 2012; 303:130-40.

18. Morinobu S, Fujimaki K, Okuyama N, Takahashi M, Duman RS. Stimulation of adenylyl cyclase and induction of brain-derived neurotrophic factor and TrkB mRNA by NKH477, a novel and potent forskolin derivative. J Neurochem. 1999; 72:2198-205. [PubMed: 10217303]

19. Pak KJ, Ostrom RS, Matsui M, Ehlert FJ. Impaired M3 and enhanced M2 muscarinic receptor contractile function in a streptozotocin model of mouse diabetic urinary bladder. Naunyn Schmiedebergs Arch Pharmacol. 2010; 381441:54.

20. Galvao J, Davis B, Tilley M, Normando E, Duchen MR, Cordeiro MF. Unexpected low-dose toxicity of the universal solvent DMSO. FASEB J. 2014; 28:1317-30. [PubMed: 24327606]

21. Sawada N, Nomiya M, Hood B, Koslov D, Zarifpour M, Andersson KE. Protective effect of a $\beta 3$ adrenoceptor agonist on bladder function in a rat model of chronic bladder ischemia. Eur Urol. 2013; 64:664-71. [PubMed: 23838637]

22. Ferguson AC, Sutton BW, Boone TB, Ford AP, Munoz A. Inhibition of urothelial $\mathrm{P} 2 \times 3$ receptors prevents desensitization of purinergic detrusor contractions in the rat bladder. BJU Int. 2015; 116:293-301. [PubMed: 25430615]

23. Hurt KJ, Musicki B, Palese MA, et al. Akt-dependent phosphorylation of endothelial nitric-oxide synthase mediates penile erection. Proc Natl Acad Sci U S A. 2002; 99:4061-6. [PubMed: 11904450]

24. Altman DG, Bland JM. Standard deviations and standard errors. BMJ. 2005; 331:903. [PubMed: 16223828]

25. Charrua A, Cruz CD, Jansen D, Rozenberg B, Heesakkers J, Cruz F. Co-administration of transient receptor potential vanilloid 4 (TRPV4) and TRPV1 antagonists potentiate the effect of each drug in a rat model of cystitis. BJU Int. 2015; 115:452-60. [PubMed: 25041193]

26. Sullivan GM, Feinn R. Using Effect Size-or Why the P Value Is Not Enough. J Grad Med Educ. 2012; 4:279-82. [PubMed: 23997866]

27. Abrams P. Describing bladder storage function: overactive bladder syndrome and detrusor overactivity. Urology. 2003; 62:28-37. [PubMed: 14662404]

28. Grassie ME, Moffat LD, Walsh MP, MacDonald JA. The myosin phosphatase targeting protein (MYPT) family: a regulated mechanism for achieving substrate specificity of the catalytic subunit of protein phosphatase type 1ठ. Arch Biochem Biophys. 2011; 510:147-59. [PubMed: 21291858]

29. Gallagher PJ, Herring BP, Stull JT. Myosin light chain kinases. J Muscle Res Cell Motil. 1997; 18:1-16. [PubMed: 9147985]

30. Lincoln TM. Myosin phosphatase regulatory pathways: different functions or redundant functions? Circ Res. 2007; 100:10-2. [PubMed: 17204659]

31. Liu B, Lee YC, Alwaal A, et al. Carbachol-induced signaling through Thr696-phosphorylation of myosin phosphatase-targeting subunit 1 (MYPT1) in rat bladder smooth muscle cells. Int Urol Nephrol. 2016; 48:1237-42. [PubMed: 27118568] 
32. Hess DT, Matsumoto A, Kim SO, Marshall HE, Stamler JS. Protein S-nitrosylation: purview and parameters. Nat Rev Mol Cell Biol. 2005; 6:150-66. [PubMed: 15688001]

33. Sopko NA, Hannan JL, Bivalacqua TJ. Understanding and targeting the Rho kinase pathway in erectile dysfunction. Nat Rev Urol. 2014; 11:622-8. [PubMed: 25311680]

34. Jin L, Liu T, Lagoda GA, Champion HC, Bivalacqua TJ, Burnett AL. Elevated RhoA/Rho-kinase activity in the aged rat penis: mechanism for age-associated erectile dysfunction. FASEB J. 2006; 20:536-8. [PubMed: 16396994]

35. Jin L, Burnett AL. RhoA/Rho-kinase in erectile tissue: mechanisms of disease and therapeutic insights. Clin Sci (Lond). 2006; 110:153-65. [PubMed: 16411892]

36. Kil IS, Lee JH, Yoon SH, et al. S-Nitrosylation of p47(phox) enhances phosphorylation by casein kinase 2. Redox Rep. 2015; 20:228-33. [PubMed: 26018922]

37. Chamseddine AH, Miller FJ Jr. Gp91phox contributes to NADPH oxidase activity in aortic fibroblasts but not smooth muscle cells. Am J Physiol Heart Circ Physiol. 2003; 285:2284-9.

38. Savchenko VL. Regulation of NADPH oxidase gene expression with PKA and cytokine IL-4 in neurons and microglia. Neurotox Res. 2013; 23:201-13. [PubMed: 22565378]

39. Sugimoto M, Nakayama M, Goto TM, Amano M, Komori K, Kaibuchi K. Rho-kinase phosphorylates eNOS at threonine 495 in endothelial cells. Biochem Biophys Res Commun. 2007; 361:462-7. [PubMed: 17651694]

40. Rajagopal S, Nalli AD, Kumar DP, et al. Cytokine-induced S-nitrosylation of soluble guanylyl cyclase and expression of phosphodiesterase 1A contribute to dysfunction of longitudinal smooth muscle relaxation. J Pharmacol Exp Ther. 2015; 352:509-18. [PubMed: 25550199]

41. Aburima A, Wraith KS, Raslan Z, Law R, Magwenzi S, Naseem KM. cAMP signaling regulates platelet myosin light chain (MLC) phosphorylation and shape change through targeting the RhoARho kinase-MLC phosphatase signaling pathway. Blood. 2013; 122:3533-45. [PubMed: 24100445]

42. Yamanishi T, Chapple CR, Yasuda K, Chess-Williams R. The role of M(2)-muscarinic receptors in mediating contraction of the pig urinary bladder in vitro. Br J Pharmacol. 2000; 131:1482-8. [PubMed: 11090124]

43. Anderson KE. Pharmacology of lower urinary tract smooth muscles and penile erectile tissues. Pharmacol Rev. 1993; 45:253-308. [PubMed: 8248281]

44. Ellerbroek SM, Wennerberg K, Burridge K. Serine phosphorylation negatively regulates RhoA in vivo. J Biol Chem. 2003; 278:19023-31. [PubMed: 12654918]

45. Jones SE, Palmer TM. Protein kinase A-mediated phosphorylation of RhoA on serine 188 triggers the rapid induction of a neuroendocrine-like phenotype in prostate cancer epithelial cells. Cell Signal. 2012; 24:1504-14. [PubMed: 22504159]

46. Chen Y, Mathias L, Falero-Perez JM, Kim SF. PKA-mediated phosphorylation of Dexras1 suppresses iron trafficking by inhibiting S-nitrosylation. FEBS Lett. 2015; 589:3212-9. [PubMed: 26358293]

47. Liu PY, Liao JK. A method for measuring Rho kinase activity in tissues and cells. Methods Enzymol. 2008; 439:181-9. [PubMed: 18374165]

48. Masumoto A, Mohri M, Shimokawa H, Urakami L, Usui M, Takeshita A. Suppression of coronary artery spasm by the Rho-kinase inhibitor fasudil in patients with vasospastic angina. Circulation. 2002; 105:1545-7. [PubMed: 11927519]

49. Wróbel A, Rechberger T. The influence of Rho-kinase inhibition on acetic acid-induced detrusor overactivity. Neurourol Urodyn. 2015 


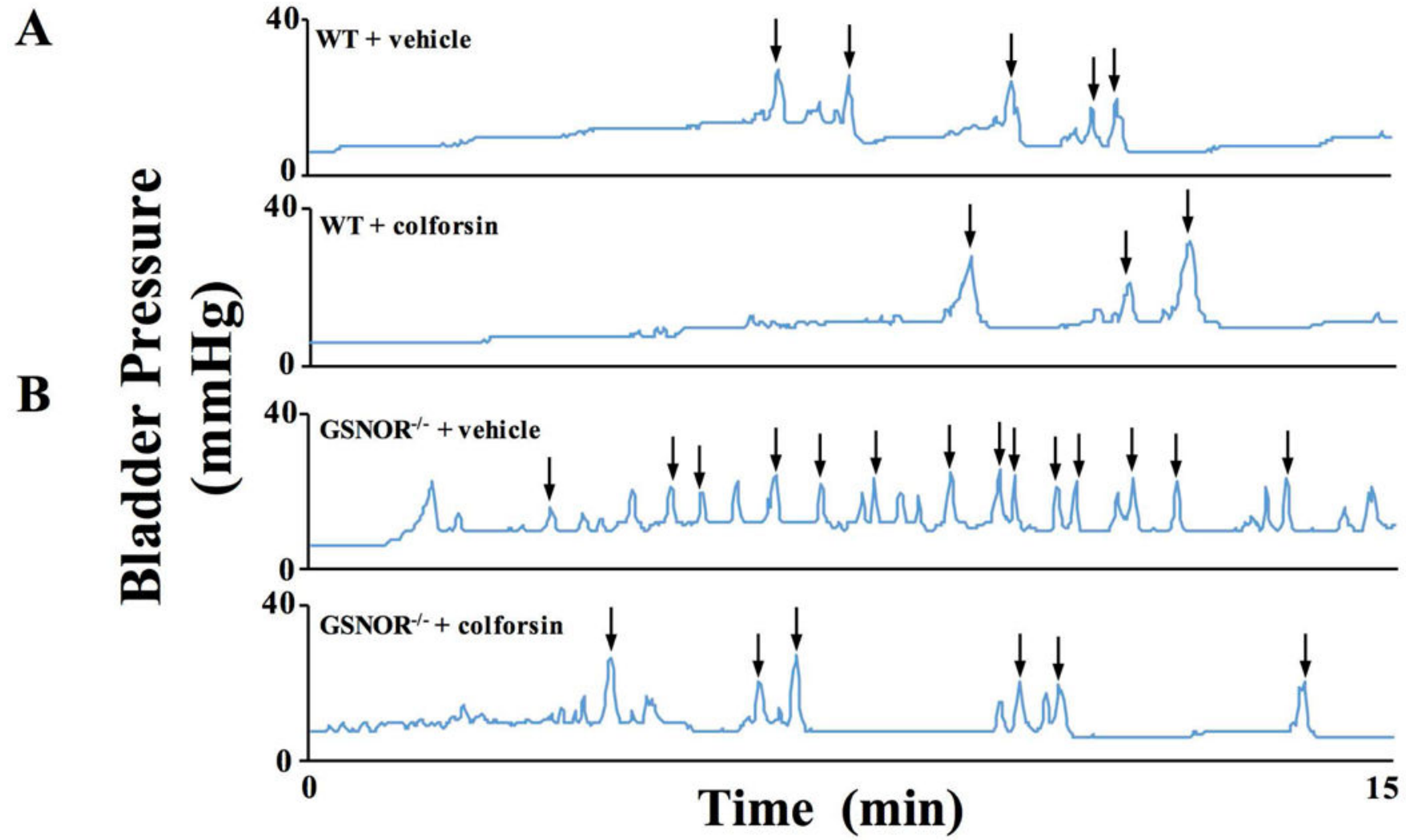

Fig. 1.

Representative cystometric traces in WT (A) treated with vehicle, WT treated with colforsin, $\mathrm{GSNOR}^{-1-}$ (B) treated with vehicle, and $\mathrm{GSNOR}^{-1-}$ treated with colforsin.

Arrows indicate voiding contractions. 

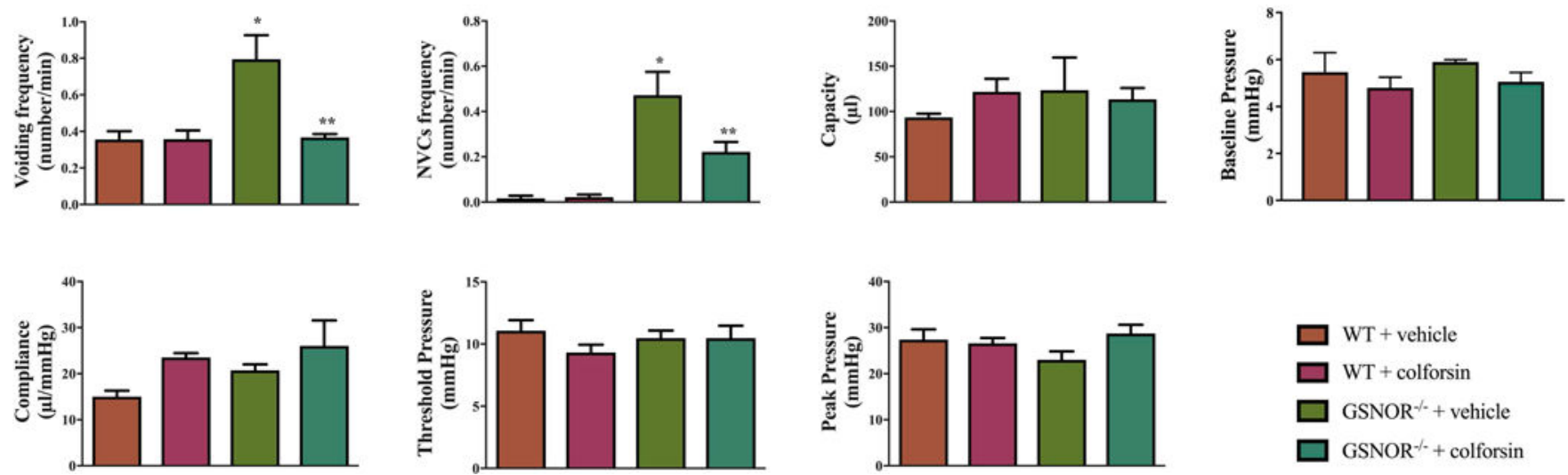

WT + vehicle

$\square \mathrm{WT}+$ colforsin

$\square \mathrm{GSNOR}^{-/ *}+$ vehicle

$\mathrm{GSNOR}^{-/}+$colforsin

Fig. 2.

Effect of colforsin on cystometric parameters (voiding frequency, non-voiding contractions [NVCs] frequency, capacity, compliance, baseline pressure, threshold pressure and peak pressure) in WT treated with vehicle, WT treated with colforsin, GSNOR ${ }^{-/}$treated with vehicle and GSNOR ${ }^{-/-}$mice treated with colforsin. Each bar represents the mean \pm SEM from six mice. $* \mathrm{P}<0.05$ versus WT treated with Vehicle. $* * \mathrm{P}<0.05$ versus $\mathrm{GSNOR}^{-/-}$treated with vehicle. 
A

B

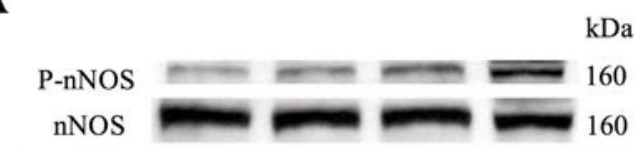

eNOS
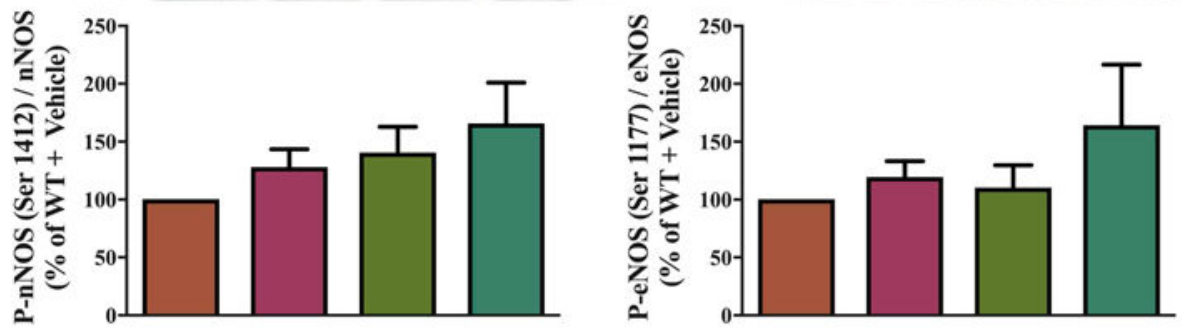

C
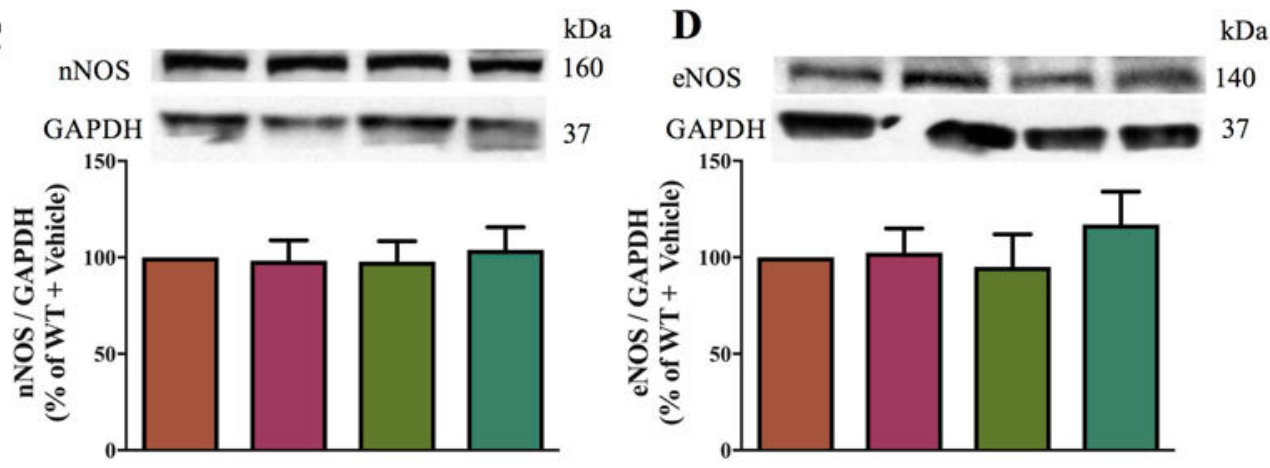

$\square$ WT + vehicle

$\square \mathrm{WT}+$ colforsin

$\square \mathrm{GSNOR}^{-/-}+$vehicle

$\mathrm{GSNOR}^{-/-}+$colforsin

Fig. 3.

Effect of colforsin treatment on protein expression levels of P-nNOS (Ser-1412) (A), P-

eNOS (Ser-1177) (B), nNOS (C) and eNOS (D) in bladders. Upper panels are representative Western immunoblots of P-nNOS/nNOS, P-eNOS/eNOS, nNOS/GAPDH and eNOS/

GAPDH in bladders of WT treated with vehicle, WT treated with colforsin, $\mathrm{GSNOR}^{-/-}$ treated with vehicle and $\mathrm{GSNOR}^{-/-}$mice treated with colforsin. Lower panels are quantitative analyses of P-nNOS/nNOS, P-eNOS/eNOS, nNOS/GAPDH and eNOS/ GAPDH in bladders of the same treatments groups. Each bar represents the mean \pm SEM from seven to nine mice. 
A
C

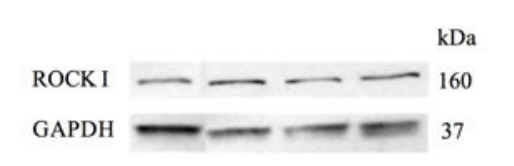

B
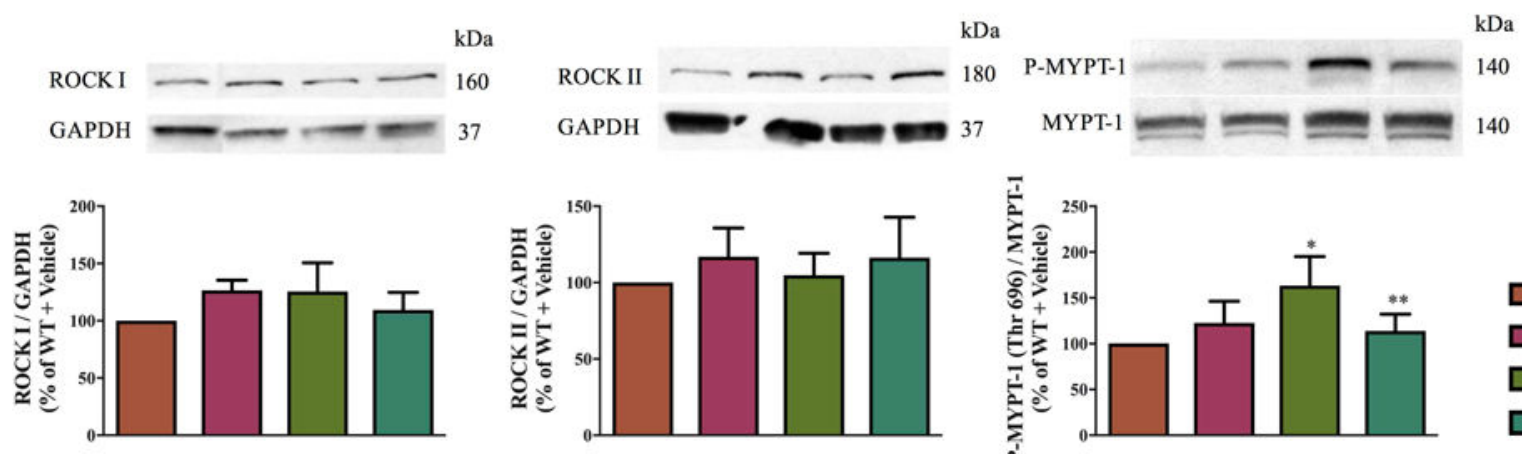

WT + vehicle

WT + colforsin

$\mathrm{GSNOR}^{-/+}+$vehicle

GSNOR $^{* *}+$ colforsin

Fig. 4.

Effect of colforsin on protein expression levels of ROCK I (A), ROCK II (B) and P-MYPT1 (Thr 696) (C). Upper panels are representative Western immunoblots of ROCK I/GAPDH, ROCK II/GAPDH, P-MYPT-1/MYPT-1 in bladders of WT treated with vehicle, WT treated with colforsin, GSNOR ${ }^{-/-}$treated with vehicle and $\mathrm{GSNOR}^{-/-}$mice treated with colforsin. Lower panels are quantitative analyses of ROCK I/GAPDH, ROCK II/GAPDH, P-MYPT-1/ MYPT-1 of the same groups. Each bar represents the mean \pm SEM from four (ROCK I), seven to nine (ROCK II) and six (P-MYPT-1) mice. ${ }^{*} \mathrm{P}<0.05$ versus WT treated with vehicle. $* * \mathrm{P}<0.05$ versus $\mathrm{GSNOR}^{-/-}$treated with vehicle. 
A
B
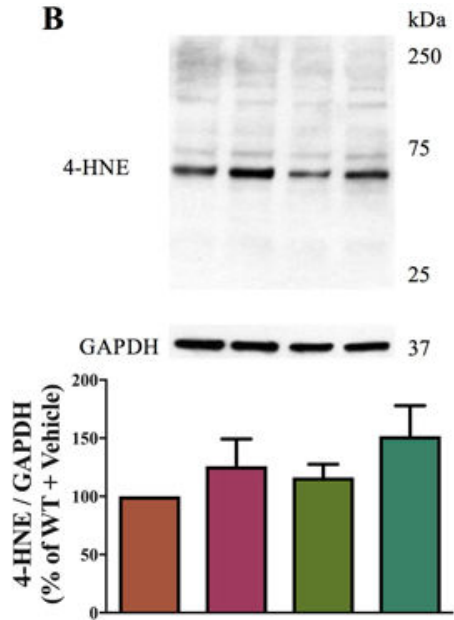

Da 75

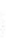
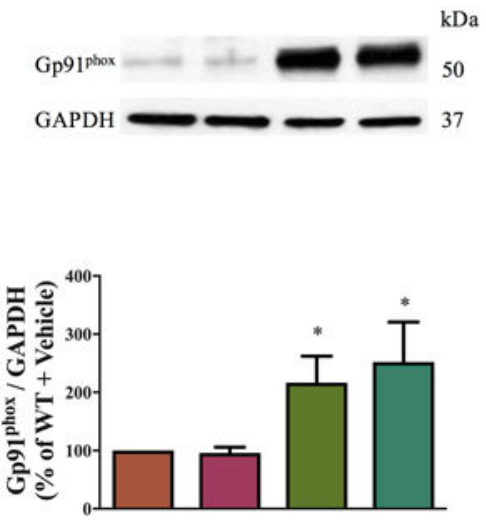
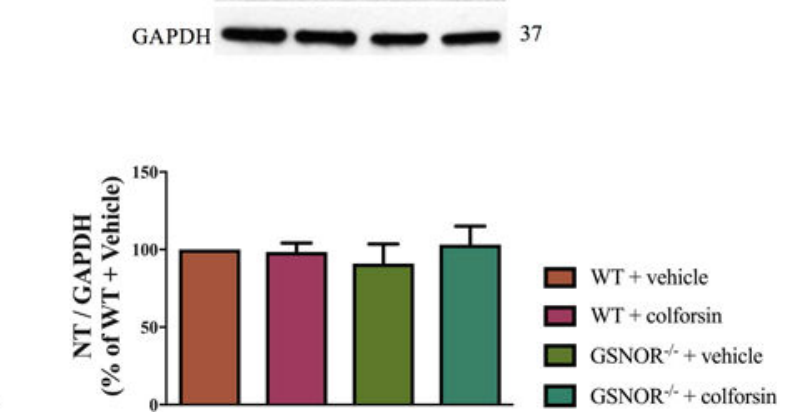

Fig. 5.

Effect of colforsin on protein expression levels of NAPDH oxidase catalytic subunit gp91 ${ }^{\text {phox }}$ (A), 4-HNE (B) and Nitrotyrosine (NT) (C) in bladders. Upper panels are representative Western immunoblots of gp91 phox/GAPDH, 4-HNE/GAPDH, NT/GAPDH in bladders of WT treated with vehicle, WT treated with colforsin, GSNOR ${ }^{-/}$treated with vehicle and $\mathrm{GSNOR}^{-/-}$mice treated with colforsin. Lower panels are quantitative analyses of gp91 ${ }^{\text {phox} / G A P D H, ~ 4 H N E / G A P D H, ~ N T / G A P D H ~ i n ~ b l a d d e r s ~ o f ~ t h e ~ s a m e ~ t r e a t m e n t ~}$ groups. Each bar represents the mean \pm SEM from seven to nine mice. $* \mathrm{P}<0.05$ versus WT treated vehicle 


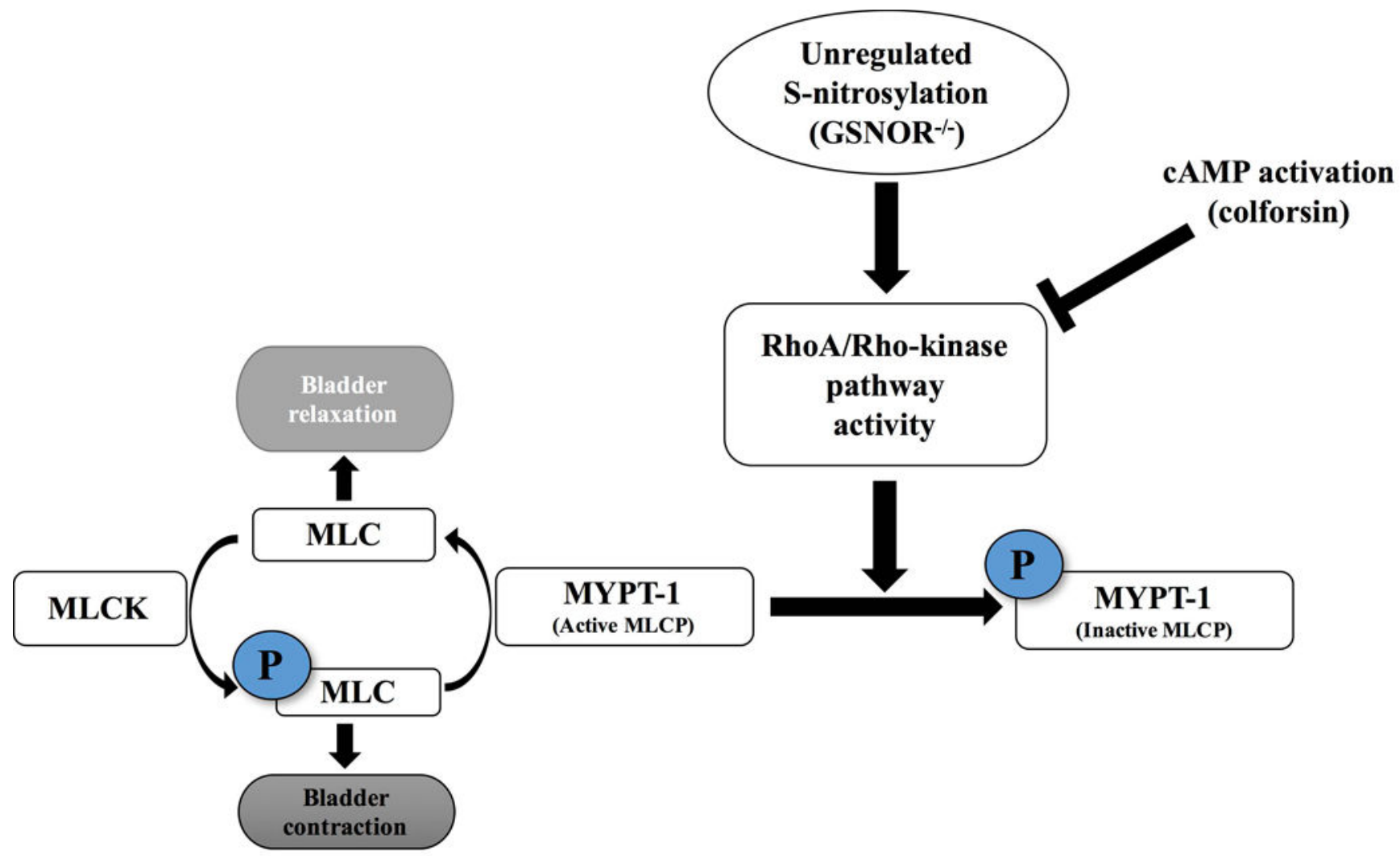

Fig. 6.

Hypothetical mechanisms involved in $\mathrm{GSNOR}^{-/-}$mouse bladder function and pharmacomodulation by colforsin. Up-regulation of RhoA/Rho-kinase pathway activity leads to excessive phosphorylation of MYPT-1 which inhibits MLCP function increasing bladder contraction. cAMP activator colforsin inhibits RhoA/Rho-kinase pathway activity which normalizes MYPT-1 phosphorylation and activates MLCP leading to bladder relaxation. 